\title{
Respiratory findings in sudden unexpected death in epilepsy: a point of convergence for clinical pulmonologists and neurologists
}

\author{
Alterações respiratórias e morte súbita em epilepsia: um ponto de convergência \\ para pneumologistas clínicos e neurologistas
}

\section{To the Editor:}

Epilepsy is the most common serious neurological disorder. Unfortunately, individuals with epilepsy are at higher risk of death than is the general population, and sudden unexpected death in epilepsy (SUDEP) is the leading cause of death directly related to epilepsy. ${ }^{(1)}$ It has been reported that SUDEP is responsible for $7.5 \%$ to $17 \%$ of all deaths in epilepsy and has an incidence among adults between 1:500 and 1:1,000 patientyears. ${ }^{(2)}$ SUDEP is defined by precise criteria approved in 1997 by an expert panel as sudden, unexpected, witnessed or unwitnessed, nontraumatic and nondrowning death in patients with epilepsy, with or without evidence of a seizure and excluding documented status epilepticus, in which postmortem examination does not reveal a toxicological or anatomical cause of death. ${ }^{(3)}$

Although results are not wholly consistent among studies, a number of factors have been reported to be associated with SUDEP. Such factors include refractoriness of the epilepsy, presence of generalized tonic-clonic seizures, polytherapy with antiepileptic drugs, younger age, duration of the seizure disorder ranging from 15 to 20 years and early onset of epilepsy. ${ }^{(1)}$ The pathophysiological mechanisms of SUDEP remain unknown. However, it is highly likely that certain pulmonary abnormalities play a role.(1) Over the past two decades, major advances have been made concerning SUDEP. However, our understanding of the exact mechanisms underlying SUDEP, as well as of the best way to prevent it, is still incomplete.

A diagnosis of SUDEP indicates that no cause of death was identified. In this sense, a better understanding of the mechanisms of SUDEP is essential. Although the cause of death in SUDEP remains unknown, a number of postmortem, ictal and interictal pulmonary changes indicate the possibility of seizure-induced pulmonary SUDEP.(1,3) Respiratory abnormalities such as pulmonary edema, central and obstructive apnea and hypoxia are well documented in patients with epilepsy. ${ }^{(1,3)}$ Following this reasoning, a number of arguments might be put forward. First, detection or exclusion of a pulmonary disease as the cause of death can be difficult even at autopsy, because forensic autopsy is performed in a considerable number of SUDEP cases..$^{(1)}$ Increased lung weight, pulmonary congestion and edema have frequently been observed in postmortem examinations of SUDEP victims. ${ }^{(4)}$ Second, seizure-associated respiratory involvement, due to apnea, could be considered a prominent feature in SUDEP cases. In an elegant study evaluating ictal cardiorespiratory changes in adults with epilepsy, ${ }^{(5)}$ apnea lasting at least 10 seconds occurred in $60 \%$ of patients and was postictal in 40\% of the recorded seizures. Although apnea was central in all 10 of the patients evaluated, obstructive apnea was observed in only 3 . The majority of studies of cardiorespiratory function have focused on changes during seizures. However, our research group recently investigated whether epilepsy influences basal respiratory parameters in patients with temporal lobe epilepsy during the interictal period. ${ }^{(6)}$ Although our study did not identify any specific respiratory abnormality in temporal lobe epilepsy patients during the interictal period, we believe that clinical data related to pulmonary disorders in individuals with epilepsy should be better investigated. ${ }^{(6)}$ Understanding the cardiorespiratory mechanism involved in SUDEP could be a key to prevention. One pathogenetic mechanism of SUDEP apnea could be epileptic activity transmitted to the cerebral respiratory centers, ${ }^{(1)}$ culminating in a lethal vicious cycle of events. ${ }^{(4)}$ It has been proposed that brainstem respiratory activity is suppressed directly by seizure activity or indirectly by hypoxia from ictal cardiac arrest. ${ }^{(4)}$

We are in total agreement with suggestions that it would be interesting to determine whether cardiorespiratory comorbidity is higher among epilepsy patients than in the general 
population, as well as whether the prevalence of cardiorespiratory comorbidity differs between epilepsy patients with and without known risk factors for SUDEP.(1) In view of the deficiencies in the current knowledge, we believe that there is an urgent need for large-scale, prospective, international, community-based studies of cardiorespiratory abnormalities in patients with epilepsy to explore risk factors in more detail so that preventive strategies can be planned. To that end, we are certain that a point of convergence between pulmonologists and neurologists could be identified within this clinical context.

Fulvio Alexandre Scorza Professor in the Department of Experimental Neurology, Universidade Federal de São Paulo/ Escola Paulista de Medicina (UNIFESP/EPM, Federal University of Sao Paulo/Paulista School of Medicine) Sao Paulo, Brazil

Vera Cristina Terra
Attending Physician in the
Neurology Department,
Centro de Cirurgia de Epilepsia
(CIREP, Epilepsy Surgical Center) of the University of São Paulo at Ribeirão Preto School of Medicine Hospital das Clínicas, São Paulo (SP) Brazil

Marly de Albuquerque Professor in the Department of Experimental Neurology, Universidade Federal de São Paulo/ Escola Paulista de Medicina (UNIFESP/EPM, Federal University of Sao Paulo/Paulista School of Medicine) Sao Paulo, Brazil

Esper Abrão Cavalheiro Professor in the Department of Experimental Neurology, Universidade Federal de São Paulo/ Escola Paulista de Medicina (UNIFESP/EPM, Federal University of Sao Paulo/Paulista School of Medicine) Sao Paulo, Brazil

\section{References}

1. Stöllberger C, Finsterer J. Cardiorespiratory findings in sudden unexplained/unexpected death in epilepsy (SUDEP). Epilepsy Res. 2004;59(1):51-60.

2. Schuele SU, Widdess-Walsh P, Bermeo A, Lüders HO. Sudden unexplained death in epilepsy: the role of the heart. Cleve Clin J Med. 2007;74 Suppl 1:S121-7.

3. Nashef $\mathrm{L}$, Hindocha N, Makoff A. Risk factors in sudden death in epilepsy (SUDEP): the quest for mechanisms. Epilepsia. 2007;48(5):859-71.

4. So EL. What is known about the mechanisms underlying SUDEP? Epilepsia. 2008;49 Suppl. 9:93-8.

5. Nashef L, Walker F, Allen P, Sander JW, Shorvon SD, Fish DR. Apnoea and bradycardia during epileptic seizures: relation to sudden death in epilepsy. J Neurol Neurosurg Psychiatry. 1996;60(3):297-300.

6 .Scorza FA, Abreu AM, Albuquerque M, Pacheco JB, Breviglieri R, Sander JW, et al. Quantification of respiratory parameters in patients with temporal lobe epilepsy. Arq Neuropsiquiatr. 2007;65(2B):450-3. 